\title{
Developmental Changes in the Superoxide Dismutase Activity of Human Skin Fibroblasts Are Maintained In Vitro and Are Not Caused by Oxygen
}

\author{
R. G. Allen and A. K. Balin \\ Laboratory for Investigative Dermatology, The Rockefeller University, New York, New York 10021
}

\begin{abstract}
Confluent cultures of human skin fibroblast lines established from fetal and postnatal donors were exposed to a broad range of oxygen tensions (10-600 $\mathrm{mmHg}$ ) for $1 \mathrm{wk}$; superoxide dismutase (SOD) activity was subsequently determined. Hyperoxia increased SOD activity slightly in postnatal lines but not in fetal lines. The magnitude of the increase in postnatal lines was not significant. Fetal lines exhibit only about one-fifth the SOD activity observed in postnatal lines. The results indicate that, while development-associated changes in SOD do occur in human cells, these alterations do not result from variations in ambient oxygen tension.
\end{abstract}

\section{Introduction}

Superoxide dismutase (SOD) ${ }^{1}$ (EC 1.15.1.1), is an enzyme that catalyzes removal of superoxide free radicals $\left(\mathrm{O}_{\overline{2}}^{\overline{2}}\right.$ from cells; it produces hydrogen peroxide and molecular oxygen. SOD activity changes during cell state transitions of various types (1). Tumor cells, which have regressed from a fully differentiated state, exhibit lower SOD activity than do healthy cells of similar tissue origin (2-6). SOD activity increases dramatically during the development of a variety of organisms belonging to phylogenetic groups as diverse as slime molds (7-11), insects (12), amphibians (13), and mammals (14-18). The underlying causes of change in SOD activity in tumor cells and during development are unclear.

It is known that elevation of ambient oxygen tension can stimulate the activity of SOD in normal, fully developed tissues (19). It has been hypothesized that removal of developing organisms from their in utero environment into the relatively high oxygen atmosphere at birth is the cause of subsequent increases in SOD activity (20-22). Consistent with this hypothesis is the observation that the activity of SOD is more readily induced by oxygen in the tissues of some developing

Address all correspondence to Dr. R. G. Allen, Laboratory for Investigative Dermatology, The Rockefeller University, 1230 York Avenue, New York, NY 10021.

Received for publication 18 March 1988 and in revised form 21 April 1988.

1. Abbreviations used in this paper: SOD, superoxide dismutase; MnSOD, mangano-isozyme SOD.

J. Clin. Invest.

(c) The American Society for Clinical Investigation, Inc. 0021-9738/88/08/0731/04 \$2.00

Volume 82, August 1988, 731-734 organisms and newborns than in adults (20-24). However, birth is a phenomenon that occurs only in higher organisms; development-associated changes in SOD activity are also observed in lower life forms not subjected to any sudden increase in ambient oxygen throughout life (7-13). The activity of SOD increases before birth in many mammalian species (15-18, 25). Furthermore, Frank et al. (21) report that SOD activity fails to change in response to oxygen in some newborn mammals. It has also been postulated that developmental changes in SOD activity are independent of ambient oxygen concentration $(18,25)$. To further study the underlying nature of development-associated alterations in SOD activity, we have determined the effects of a 1-wk exposure to different oxygen concentrations ranging from 10 to $600 \mathrm{mmHg}$ on the SOD activity of cultured human skin fibroblasts obtained from donors whose ages ranged from fetal to nonagenarian. The results of this study demonstrate that developmental differences exist in the SOD activity of skin fibroblasts derived from fetal and postnatal humans and that these differences are maintained in culture independently of ambient oxygen tension.

\section{Methods}

Details of the cell cultivation procedures have been described previously $(26,27)$. Cells were grown without shaking in DME with 1 $\mathrm{g} /$ liter glucose (Gibco Laboratories, Grand Island, NY), supplemented with $2 \mathrm{mM}$ L-glutamine, and 10\% (vol/vol) FBS. Antibiotics were not used except in the initial outgrowth from primary cultures. All cultures employed for experimentation were free of mycoplasma contamination. Human skin fibroblasts cultures were established from skin samples obtained from fetal, young, and old donors; the ages of the postnatal donors employed in this study ranged from 4 to $98 \mathrm{yr}$. In some individuals, biopsies were obtained from the upper outer quadrant of the buttock, other cultures were established from normal skin excised as Burrow's triangles during repair of surgical procedures. Additionally, AG7725, AG4059, AG6234, AG6291, and AG6308 were obtained from the National Institute on Aging cell repository. Fetal skin fibroblast cultures (AG4431, AG4525, AG4449, AG4392, AG4451, AG6561, AG6557, and AG6559) were obtained from the National Institute on Aging cell repository at the Institute for Medical Research, Camden, NJ. During the initial phase when the cell lines were expanded, the cultures were inoculated into polystyrene flasks at $10^{4}$ cells $/ \mathrm{cm}^{2}$. After the cultures had become confluent (7-d growth) the flasks were refed with fresh medium that contained $0.5 \%$ FBS. The flasks were equilibrated with an atmosphere that contained $0,5,20,35$, 50 , or $95 \% \mathrm{O}_{2}, 5 \% \mathrm{CO}_{2}$, where the balance was made up by $\mathrm{N}_{2}$, and then they were incubated at $37^{\circ} \mathrm{C}$ for an additional $7 \mathrm{~d}$. Immediately before harvest the oxygen tension of the medium was determined using a blood gas analyzer as previously described $(26,27)$. Cells were harvested by trypsinization, washed three times with PBS and frozen under liquid nitrogen until measurements were made. Although tryp- 
sinization of cells has been observed to decrease SOD activity in some cases (28), we have found that neutralization of trypsin with soya bean trypsin inhibitor (Sigma Chemical Co., St. Louis, MO) prevents any loss of SOD activity during harvest.

The positive assay of Misra and Fridovich (29) was used to quantify the activity of SOD isozymes as previously described (30). Units of activity were determined by standardizing this procedure with samples of commercially prepared SOD, the activity of which was measured with the cytochrome $c$ procedure (31). The mangano-isozyme (MnSOD) was determined with the above procedure except that $2 \mathrm{mM}$ $\mathrm{KCN}$ was added to the 0 -dianisidine solution.

\section{Results and Discussion}

Total SOD activity and the activity of the MnSOD and $\mathrm{Cu} / \mathrm{Zn}$ isozymes were determined within several weeks of the date of harvest. Although SOD activity was more variable in the postnatal group than in the fetal group, as indicated by larger standard deviations (Fig. 1), no age-associated trends in SOD activity were observed in the postnatal lines. Also, no age-dependent differences in variance were observed when the data from young and old postnatal donors were compared. Since the activity of SOD, determined in cells from young and old postnatal donors, was not statistically different, these data were averaged into a single group (postnatal group) in this report. Exposure of human skin fibroblasts, derived from postnatal donors, to either 50 or $95 \%$ oxygen for $7 \mathrm{~d}$ induced an increase in total SOD (Fig. $1 A$ ) and in MnSOD (Fig. $1 B$ ) as compared with the SOD activity found at $0 \%$ oxygen. This stimulation occurred in all 14 of the postnatal lines employed for study and thus represents a statistically significant trend (Runs test $P<0.001$ ); however, a comparison of high and low average SOD activities revealed that the magnitude of the increase was not significant. No stimulation of total SOD or $\mathrm{MnSOD}$ was observed in fetal lines. $\mathrm{Cu} / \mathrm{Zn}$ SOD activity was stimulated by oxygen in both fetal and postnatal lines, but the magnitude of the stimulation was too small to be significant. These results indicate that induction of SOD activity by $\mathrm{O}_{2}$ is of limited magnitude in both fetal and postnatal lines of skin fibroblasts.

In contrast to the modest ability of oxygen to induce SOD in human skin fibroblasts, development exerts a profound influence on SOD activity. Total SOD activity (Fig. $1 \mathrm{~A}$ ) was lower in fetal lines than in postnatal lines (ANOVA, $P<0.01$ ). This difference was due to MnSOD activity (Fig. $1 B$ ), which was significantly lower in the fetal lines (ANOVA, $P<0.01$ ). $\mathrm{Cu} / \mathrm{Zn}$ SOD activity was similar in fetal and postnatal lines (Fig. 1C). Comparison of data presented in Fig. 1, $A$ and $C$ reveals that in fetal lines, $\mathrm{Cu} / \mathrm{Zn}$ SOD is $55 \%$ of the total SOD activity, whereas $\mathrm{Cu} / \mathrm{Zn}$ SOD activity is only $17 \%$ of total SOD activity in postnatal lines. These results demonstrate marked developmental changes in MnSOD activity and indicate that, in human skin fibroblasts, the changes are not the result of altered ambient oxygen tension. The relatively greater variation in SOD activity observed in the postnatal group as compared with the fetal group suggests that the magnitude of development-associated change in SOD activity is subject to individual variation.

Few investigators have examined whether developmentassociated increases in SOD were the result of changes in only one isozyme. Tanswell and Freeman (18) reported that in newborn rats MnSOD did not exhibit a large, rapid increase
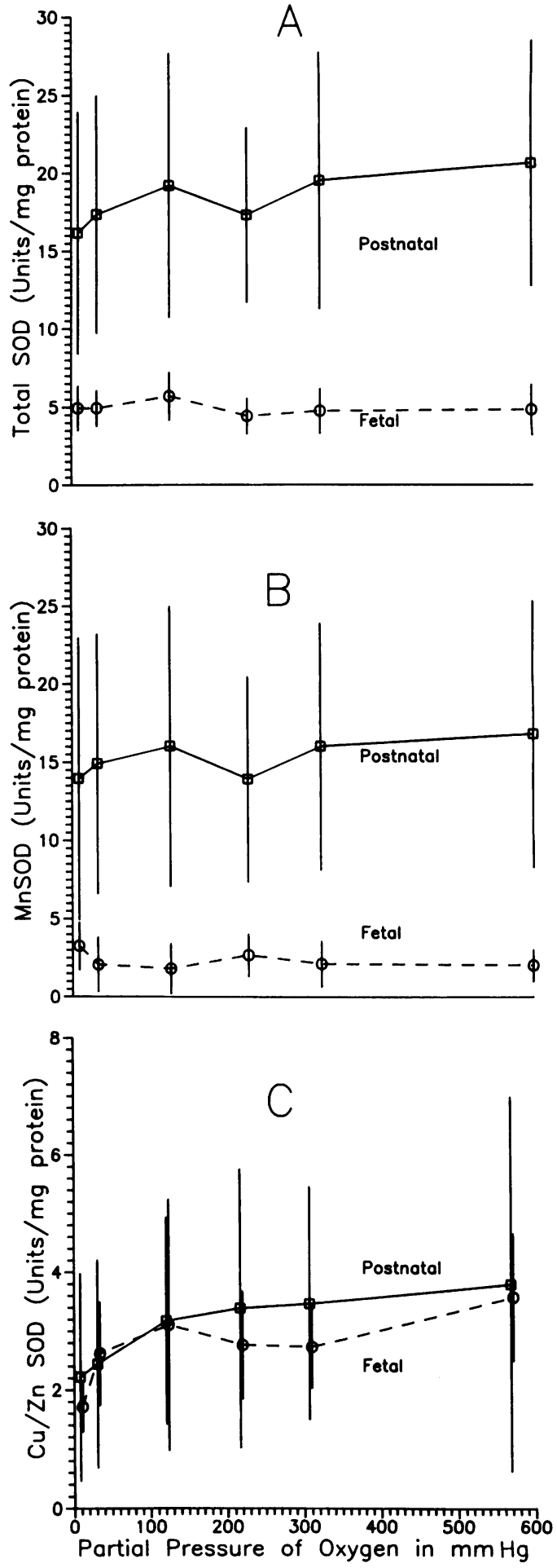

Figure 1. SOD activity in fetal and postnatal lines of human skin fibroblasts exposed to various oxygen tensions. (A) Total SOD; $(B)$ $\mathrm{MnSOD;}(C) \mathrm{Cu} / \mathrm{Zn}$ SOD. Fetal, $n=7$; postnatal, $n=14$.

whereas $\mathrm{Cu} / \mathrm{Zn}$ SOD did. In organisms such as mice, rabbits, and rats, oxygen can rapidly induce total SOD activity (21). Conversely, Frank et al. (21), found that newborn guinea pigs and hamsters cannot increase their SOD activity rapidly and therefore succumb to hyperoxia. Frank et al. (21) suggested that the ability to respond to oxidative stress may itself be 
related to the stage of development. They further postulated that guinea pigs and hamsters, which are more developed at the time of birth than rats, rabbits, or mice, are past the developmental stage at which SOD may be induced. The human fetal cell lines used in this study were obtained from individuals not more than 17 wk gestational age; thus, if such a phase of rapid SOD induction exists in humans, it would be quite early in their developmental history. It would also seem relevant that SOD activity is not induced by hyperoxia in human fetal lung fibroblasts after a 48-h exposure and that the level of activity we previously observed in fetal lung fibroblast corresponds to the activity in fetal skin fibroblasts that we report here (32).

The underlying cause of developmental changes in SOD activity is not clear. Mitochondrial number increases in mammalian cells during development (33). It is, therefore, possible that development-associated differences in MnSOD activity observed here are due to differences in the number of mitochondria. However, in Physarum, MnSOD activity increases by as much as 46 -fold during differentiation, whereas, the number of mitochondria either remains constant, or decreases during this process (7). Furthermore, in insects the increase in MnSOD activity observed during metamorphosis is far greater than can be explained by changes in mitochondrial number (34). It has been postulated that variations in the level of endogenous oxidative stress are responsible for changes in SOD activity observed during development (1). Although the range of oxygen tensions used in this study exerts a strong influence on a wide variety of physiological and biochemical parameters $(26,27,32)$, it induced no significant increase in the SOD activity of human skin fibroblasts. The failure of elevated oxygen tensions to significantly alter SOD activity in fetal and postnatal lines may indicate that the influence of hyperoxia on these cells is largely mediated by factors other than $\mathrm{O}_{\overline{2}}$ generation. However, the failure of hyperoxia to induce SOD activity does not rule out the possibility that the rate of superoxide radical generation is different in fetal and postnatal lines.

As noted above, many tumor cells exhibit low SOD activity; it has also been observed that the lower activity in transformed cells often results from loss of MnSOD (2-6). It has been postulated that loss of MnSOD activity plays a causal role in cellular transformation $(4,5)$. We recently demonstrated that liposomal addition of SOD to a nondifferentiating strain of Physarum induces differentiation in the organism (35). Whether development-associated changes in SOD activity in mammalian cells have any influence on gene expression is presently unclear. Further study is required to determine the precise cause of development-associated differences in SOD activity and whether these changes exert any effects on the expression of other genes.

The results of this study reveal that developmental variations in SOD expression are maintained in vitro. On the basis of this study, we infer that development-associated alterations in SOD activity in human cells are not due to changes in the ambient oxygen concentration.

\section{Acknowledgments}

The expert technical assistance of Mrs. Ina Leong, Miss Wende Reenstra, Mr. Richard Reimer, and Miss Anitha Mathew is gratefully acknowledged. We thank Drs. D. Martin Carter, Janet Moy, and Andrew Lin for remarks helpful in the preparation of this report.
Support for this work was provided by National Institutes of Health grants AG-00282-01, AG-04993-01, AG-05346-01, RR-00102, and general support from the Pew Trust.

\section{References}

1. Sohal, R. S., R. G. Allen, and C. Nations. 1986. Oxygen free radicals play a role in cellular differentiation: an hypothesis. J. Free Radicals Biol. \& Med. 2:175-181.

2. Dionisi, O., T. Galleotti, T. Terranova, and A. Azzi. 1975. Superoxide radicals and hydrogen peroxide formation in mitochondria from normal and neoplastic tissues. Biochim. Biophys. Acta. 403:292300.

3. van Balgoony, J. N. A., and E. Roberts. 1979. Superoxide dismutase in normal and malignant tissues in different species. Comp. Biochem. Physiol. 62:263-268.

4. Oberley, L. W. 1982. Superoxide dismutases in cancer. In Superoxide Dismutase. Vol. 2. L. W. Oberley, editor. CRC Press, Boca Raton, FL. 127-165.

5. Oberley, L. W., T. D. Oberley, and G. R. Buettner. 1980. Cell differentiation and cancer: the possible role of superoxide and superoxide dismutases. Med. Hypotheses. 6:249-268.

6. Oberley, L. W., and T. D. Oberley. 1984. The role of superoxide dismutase and gene amplification in carcinogenesis. J. Theor. Biol. 106:403-422.

7. Allen, R. G., R. K. Newton, R. S. Sohal, G. L. Shipley, and C. Nations. 1985. Alterations in superoxide dismutase, glutathione, and peroxides in the plasmodial slime mold Physarum polycephalum during differentiation. J. Cell. Physiol. 125:413-419.

8. Lott, T., S. Gorman, and J. Clark. 1981. Superoxide dismutase in Didymium iridis characterization of changes in activity during senescence and sporulation. Mech. Ageing Dev. 17:119-130.

9. Allen, R. G., K. J. Farmer, R. K. Newton, R. S. Sohal, and C. Nations. 1985. Involvement of glutathione in the differentiation of the slime mold, Physarum polycephalum. Dev. Growth \& Differ. 27:615620 .

10. Allen, R. G., R. K. Newton, K. J. Farmer, and C. Nations. 1985. Effect of the free radical generator paraquat on differentiation, superoxide dismutase, glutathione, and inorganic peroxides in microplasmodia of Physarum polycephalum. Cell Tissue Kinet. 18:623-630.

11. Nations, C., R. G. Allen, K. Farmer, P. L. Toy, and R. S. Sohal. 1986. Superoxide dismutase activity during the plasmodial life cycle of Physarum polycephalum. Experientia (Basel). 42:64-66.

12. Massie, H. P., V. R. Aiello, and T. R. Williams. 1980. Changes in superoxide dismutase activity and copper during development and aging in the fruit fly Drosophila melanogaster. Mech. Ageing Dev. 12:279-286.

13. Barja Quiroga, G., and P. Gutierrez. 1984. Superoxide dismutase during the development of two amphibian species and its role in hyperoxic tolerance. Mol. Physiol. 6:221-232.

14. Mavelli, I., A. Rigo, R. Federico, M. R. Ciriolo, and G. Rotilio. 1982. Superoxide dismutase, glutathione peroxidase and catalase in developing rat brain. Biochem. J. 204:535-540.

15. Gerdin, E., O. Tyden, and U. J. Errikson. 1985. The development of antioxidant enzymic defense in the perinatal rat lung: activities of superoxide dismutase, glutathione peroxidase, and catalase. $\mathrm{Pe}$ diatr. Res. 19:687-691.

16. Russanov, E. M., M. D. Kirkova, M. S. Setchenska, and H. R. V. Arnstein. 1981. Enzymes of oxygen metabolism during erythrocyte differentiation. Biosci. Rep. 1:927-931.

17. van Hien, P., K. Kovacs, and B. Matkovics. 1974. Properties of enzymes. I. Study of superoxide dismutase activity changes in human placenta of different ages. Enzyme. 18:341-347.

18. Tanswell, A. K., and B. A. Freeman. 1984. Pulmonary antioxidant enzyme maturation in the fetal and neonatal rat. I. Developmental profiles. Pediatr. Res. 18:871-874.

19. Crapo, J. D., and D. F. Tierney. 1974. Superoxide dismutase and pulmonary oxygen toxicity. Am. J. Pathol. 226:1401-1407. 
20. Autor, A. P., L. Frank, and R. J. Roberts. 1976. Developmental characteristics of pulmonary superoxide dismutase: relationship to idiopathic respiratory distress syndrome. Pediatr. Res. 10:154-158.

21. Frank, L., J. R. Bucher, and R. J. Roberts. 1978. Oxygen toxicity in neonatal and adult animals of various species. J. Appl. Physiol. Respir. Environ. Exercise Physiol. 45:699-704.

22. Bucher, J. R., and R. J. Roberts. 1981. The development of the newborn rat lung in hyperoxia: a dose response study of lung growth maturation, and changes in antioxidant enzyme activities. Pediatr. Res. 15:999-1008.

23. Yam, J., L. Frank, and R. J. Roberts. 1978. Oxygen toxicity: comparison of biochemical responses in neonatal and adult rats. $\mathrm{Pe}$ diatr. Res. 12:115-119.

24. Warshaw, J. B., C. W. Wilson, K. Saito, and R. A. Prough. 1985. The response of glutathione and antioxidant enzymes to hyperoxia in developing lung. Pediatr. Res. 19:819-823.

25. Frank, L., and E. E. Gloseclose. 1984. Preparation for birth into an $\mathrm{O}_{2}$-rich environment: the antioxidant enzymes in the developing rabbit lung. Pediatr. Res. 18:240-244.

26. Balin, A. K., A. J. Fisher, and D. M. Carter. 1984. Oxygen modulates the growth of cells at physiological partial pressures. J. Exp. Med. 160:152-166.

27. Balin, A. K., D. B. P. Goodman, H. Rasmussen, and V. J. Cristofalo. 1976. The effects of oxygen on growth and metabolism of WI-38 cells. J. Cell. Physiol. 89:235-250.

28. Yamanaka, N., and D. Deamer. 1974. Superoxide dismutase activity in WI-38 cell cultures: effects of age, trypsinization and SV-40 transformation. Physiol. Chem. Phys. 6:95-106.

29. Misra, H. P., and I. Fridovich. 1977. Superoxide dismutase a photochemical augmentation assay. Arch. Biochem. Biophys. 181:308-312.

30. Nations, C., R. G. Allen, A. K. Balin, R. J. Reimer, and R. S. Sohal. 1987. Superoxide activity and glutathione concentration during the calcium-induced differentiation of Physarum polycephalum. $J$. Cell. Physiol. 133:181-186.

31. McCord, J. M., and I. Fridovich. 1969. Superoxide dismutase. J. Biol. Chem. 244:6049-6055.

32. Balin, A. K., R. G. Allen, and R. J. Reimer. 1988. Human fibroblast antioxidant defense response to alteration in oxygen tension. In Oxygen Radicals in Biology and Medicine. Plenum Press, New York. In press.

33. Jones, C. T., and T. P. Rolph. 1985. Metabolism during fetal life: a functional assessment of metabolic development. Physiol. Rev. 65:357-430.

34. Fernandez-Souza, J., and A. M. Michelson. 1976. Variation in superoxide dismutases during the development of the fruitfly, Ceratitis capitata. Biochem. Biophys. Res. Commun. 73:217-223.

35. Allen, R. G., A. K. Balin, R. J. Reimer, R. S. Sohal, and C. Nations. 1988. Superoxide dismutase induces differentiation in microplasmodia of the slime mold, Physarum polycephalum. Arch. Biochem. Biophys. 261:205-211. 